\title{
DEMAND-SHIFTING USING MODEL-ASSISTED CONTROL
}

\author{
Giorgos D. Kontes ${ }^{*, \dagger}$, Giorgos I. Giannakis*, Elias B. Kosmatopoulos ${ }^{* *,}$, , Dimitrios V. Rovas ${ }^{*}$, \\ *Department of Production Engineering \& Management, Technical University of Crete, Greece \\ ** Department of Electrical \& Computer Engineering, Democritus University of Thrace, Greece \\ ${ }^{\dagger}$ Informatics \& Telematics Institute, Centre for Research \& Technology-Hellas, Thessaloniki, Greece \\ ${ }^{\S}$ Correspondence author. Tel: +30-28210-37365, Email: rovas@dpem.tuc.gr
}

\begin{abstract}
Increasing energy demand and more strict environmental regulations have enabled the transition from traditional electric grids, in which centralized power plants directly transmit energy to consumers, to smart electrical grids where the existing power grid is enhanced by distributed, smallscale renewables-based energy generation systems. The use of renewables into the grid inserts uncertainty to the system, due to their stochastic output profile which strongly depends on local weather conditions and their inability to cover the total electric demand at peak demand periods. One of the most common and effective methods for tackling the peak demand period problem in buildings is the use of electricity tariffs with Time-Of-Use charges, which are based on higher energy rates during high demand periods, in order to encourage electricity load shifting from peak-demand periods to periods with lower demand. In the present work, we assume that a day-ahead notification on a (hypothetical) tariff plan is provided, and the Building Energy Management System is requested to adapt to the new setting. A photovoltaic panel is assumed available, but there is no energy storage and selling-back energy to the grid is not allowed. This way, the controller is forced to operate the actuating components of the building during the production of renewable energy, while saving energy the remaining time. A model-assisted control design methodology, utilizing a stochastic optimization algorithm and weather and occupancy predictions, is used to produce a control strategy which exploits the energy produced by the photovoltaic panel, to minimize the cooling cost of a building, for a given summer day, while maintaining user comfort at acceptable levels. The proposed approach results to substantial cost reductions when compared - for a hypothetical tariff scheme to a widely-used, static rule-based control scheme.
\end{abstract}

\section{INTRODUCTION}

Increasing energy demand and more strict environmental regulations have enabled the transition from traditional electric grids, in which centralized power plants transmit energy to the users directly, to smart electrical grids [Massoud et al. 2005, Hashmi et al. 2011], where the existing power grid is enhanced by distributed, small-scale renewables-based energy generation systems, such as photovoltaic (PV) panels, wind turbines and hydroelectric factories, to name but a few. Buildings, under the smart grid perspective are no longer isolated, but are considered interconnected entities which adaptively interact [Rokach 2012], or even active members of the grid, contributing excess energy produced (for the case of positive-energy buildings) [Gamauf et al. 2011].

The use of renewables into the grid inserts uncertainty to the system, due to their stochastic output profile which strongly depends on local weather conditions. Moreover, at peak demand periods the renewable energy production can be used to cover only part of the demand and the need to use expensive electric energy generation capacities, such as gas power plants, emerges. There has been 
significant research effort towards the development of grid management techniques and renewable incorporation approaches, in order to overcome these downsides, in literature - see [Azzopardi et al. 2009, Oyarzabal et al. 2005, Qi et al. 2012 and Lu et al. 2011] and the references therein.

One of the most common and effective methods for tackling the peak demand period problem in buildings is the use of electricity tariffs with Time-Of-Use charges, which are based on higher energy rates during high demand periods, in order to encourage electricity load shifting from peak-demand periods to periods with lower demand - see for example [Oldewurtel et al. 2010, Oldewurtel et al. 2011b, Ma et al. 2010, Ma et al. 2011, Van Staden et al. 2011, Zong et al. 2012, Gamauf et al. 2011, Qi et al. 2012 and Lu et al. 2011] and their references. This is a way of assessing the impact of the final users on the whole energy market and on the environment, while acknowledging limitations such as energy resource scarcity and finite capacities of the electricity grid.

The load shifting approach, combined with building-dedicated renewables (e.g. photovoltaic panels installed on the roof) paves the way towards parsimonious energy use and grid utilization, since the building is forced to consume as much power as possible from locally-installed renewables during peak demand periods and restrict power use from the grid only during off-peak periods. The desired behavior can be achieved by designing Building Energy Management Systems (BEMS), targeted at minimizing the total operational cost of the building, taking into account the pricing of energy, rather than minimizing the total energy consumption [e.g. Oldewurtel et al. 2011b].

In the present work, a detailed thermal simulation model of a test building, equipped with a photovoltaic system is available and a day-ahead notification on a (hypothetical) tariff plan is provided [IEA 2007]. The power produced by the PV cannot be stored, selling-back to the grid is not allowed, and each day, weather and occupancy forecasts for the following day are provided. A stochastic optimization algorithm, taking into account: a) the renewables production - as estimated using the weather forecasts; - b) the thermal behavior of the building — using the detailed thermal simulation model, along with the weather forecasts and occupancy predictions - , and; c) the current electricity tariffs, is developed to produce a control strategy to be applied in the building the following day. This strategy operates the actuating components of the building preferably during periods when renewable energy is available, while saving energy the rest of the time. This way, every day the proposed modelbased algorithm automatically shifts building demand to the time-window where energy is (forecasted to be) available from the PV array, thereby reducing resilience on grid-supplied energy.

The proposed approach is a method for automatically designing Building Energy Management Systems optimized for a specific building and targeted to the microclimate conditions of each area, maximizing the renewables energetic benefits. In the present work, an example building located in Athens, Greece is used, but the generality of the method allows its use in any building and in any test area, regardless of construction, geographical and climatic variations.

\section{METHODOLOGICAL APPROACH}

We assume that a detailed thermal simulation model of the building is available and that weather forecasts for the target area and predictions for the behavior of the buildings occupants are provided. A model-assisted control approach is applied, where a stochastic optimization algorithm evaluates a series of candidate controllers, using the simulated model of the building in order to predict the building states, for a predefined time-period. A co-simulation set-up allows the application of the dynamical actuator schedules created by the candidate controllers to be applied and evaluated to the simulated model of the building. 
Building Thermal Simulation Models Buildings are complex systems exposed to various timedependent, climatic and user induced influences. A building might consist of the building envelope, windows, glazed facade, shading devices, active and passive solar elements, heating- cooling and airsupply systems, electrical systems, lighting systems and various control systems. Building thermal simulation models are commonly used in engineering and science since they describe the dynamic behavior of the system without necessitating experimental measurements from the physical building. Thus, simulations are orders of magnitude faster and less expensive than experiments. In order to perform a simulation, the design of a mathematical model, capturing the real system's features, is required.

With respect to building thermal simulation models, a typical modeling approach is to separate the building spaces in one or more thermal zones that are coupled with each other and with the environment. A zone consists of an air volume of a uniform temperature and all surfaces bounding or inside that air volume. Energy systems are modeled independently and a coupled through the effect the have on the thermal zones. The basis for the zone air temperature calculation is the formulation of energy and moisture balances for the zone and the solution of the resulting ordinary differential equations. The formulation of the solution scheme starts with the heat balance on the zone air:

$$
\begin{aligned}
C_{z} & \frac{d T_{z}}{d t}=\sum_{i=1}^{N_{s i}} \dot{Q}_{i}+\sum_{i=1}^{N_{\text {surfaces }}} h_{i} A_{i}\left(T_{s i}-T_{z}\right) \\
& +\sum_{i=1}^{N_{z o n e s}} \dot{m}_{i} C_{p}\left(T_{z i}-T_{z}\right)+\dot{m}_{\text {inf }} C_{p}\left(T_{\infty}-T_{z}\right)+\dot{Q}_{s y s}
\end{aligned}
$$

In this equation, we have:

$$
\begin{array}{ll}
\sum_{i=1}^{N_{s i}} \dot{Q}_{i} & \text { is the sum of the convective internal loads; } \\
\sum_{\substack{i=1 \\
N_{\text {surfaces }}}}^{N_{z \text { ones }}} h_{i} A_{i}\left(T_{s i}-T_{z}\right) & \text { is the convective heat transfer from the zone surfaces; } \\
\sum_{i=1}^{\dot{m}_{\text {inf }} C_{p}\left(T_{\infty}-T_{z}\right)} \dot{m}_{i} C_{p}\left(T_{z i}-T_{z}\right) & \text { is heat transfer due to inter-zone air mixing; } \\
\dot{Q}_{\text {sys }} & \text { is heat transfer due to infiltration of outside air; } \\
C_{z} \frac{d T_{z}}{d t} & \text { is the energy stored in zone air }
\end{array}
$$

This forms essentially the energy conservation equation in each zone. Similarly for the humidity variable a second mass conservation equation is formed and solved. This set of equations, along with differential equations for all the necessary components for detailed building thermal simulation (like humidity ratio calculations) is included in intergraded toolboxes for building simulation. In the present work, one such a simulator is used (EnergyPlus) [Crawley et al. 2001], but others like [TRNSYS Klein et al. 1976] or Modelica [Haase et al. 2007] can be used. Although there are different models that can be potentially used in every simulation environment these toolboxes have been extensively tested and validated against benchmark (e.g. the BESTEST) or measurements in real buildings. 
EnergyPlus provides such an integrated toolbox. At each time step, of the solution period, first the various terms in the equation above are formed, and then the continuous equation is discretized so that it can be numerically integrated, with a time step $(\delta \mathrm{t})$ that varies typically from 10 minutes to 1 hour. A finite-difference approximation is used to discretize the heat and mass balance equations. In the simplest form a first-order approximation (the Euler formula) is used:

$$
\frac{d T_{z}}{d t}=(\delta t)^{-1}\left(T_{z}^{t}-T_{z}^{t-\delta t}\right)+O(\delta t)
$$

But in more complex cases, where the systems in the buildings have complex dynamics the use of a higher order Predictor/Corrector discretization scheme, is favored. EnergyPlus uses such a scheme and to calculate the actual zone mean air temperature at each time step first a prediction of the air system load needed to reach the desired temperature (predictor) is performed, then a simulation of the air system is done to determine its actual capability (corrector), and finally the zone air heat balance to calculate the actual zone mean air temperature is done. The following steps summarize the Predictor/Corrector process:

1. The air system energy required to balance the equation (1) is estimated, given that the zone air temperature is equal to the set-point temperature (defined by a zone thermostat).

2. Having that quantity as a demand, the air system is simulated and its actual supply capability at the time of the simulation is determined.

3. According to the selected solution algorithm, the corresponding equation is used to calculate the resulting zone temperature, where $\dot{Q}_{s y s}$ is equal to the actual air system capability, which have been estimated by step 2 .

The Control Problem The application of the finite-difference approximator (2) yields a discretization of the differential equation. The resulting system of equations is a non-linear, discrete, time-varying state-space model capable of describing in detail the dynamics of the building. Introducing a equipartitioning in the continuous time $t \in\left[0, T_{f}\right]$ of step $\delta \mathrm{T}$, and defining an index $\tau \in\left\{0,1, \ldots, T_{f}\right\}$ to the new discrete-time partitions, a more compact representation of the building thermal model, which obviously includes all physical modeling assumptions and details, is defined:

$$
x_{\tau+1}=f_{\tau}\left(x_{\tau}, u_{\tau}\right), \quad \forall \tau \in\left\{0,1, \ldots, T_{f}\right\}
$$

In this equation, $\mathrm{x}_{\tau} \in \mathbb{X}$ are the states, $\mathrm{u}_{\tau} \in \mathbb{U}$ are the actions, $\tau$ is the time-index and $\mathbb{X} \in \mathbb{R}^{\mathrm{m}}$ and $\mathbb{U} \in \mathbb{R}^{\mathrm{n}}$ are constrained sets. The state variables are the smallest possible subset of system variables that can represent the entire state of the system at any given time and, in our case, include the building states (like wall and air temperatures, humidity, etc.) and the weather conditions. The vector $\mathrm{x}_{\tau}$ containing the state variables at any given time is called the state vector. Similarly, the input vector $u_{\tau}$ contains the control inputs applied to the building (heating and cooling loads, shading angle, etc.). The state-space representation (3) is a mathematical model of the physical system as a set of input $u_{\tau}$, output $x_{\tau+1}$ and state $x_{\tau}$ variables related by first-order differential equations.

Having the model of the process at hand, the goal is to discover a series of inputs $u_{\tau}$ that lead the system to a set of states $x_{\tau}$ which are optimal with respect to a performance index $J$. This index is called the cost function and is defined as:

$$
J_{\tau}=J_{\tau}\left(x_{\tau}, u_{\tau}\right), \quad \forall \tau \in\left\{0,1, \ldots, T_{f}\right\}
$$

$x_{\tau}$ and $u_{\tau}$ here are the same as in (3). The intuition behind the cost function can be apprehended with the help of a simple example: assume a simple one-room building equipped with an Air-Conditioning (AC) system, located in Athens, Greece; every day during summer period a series of set-points of the AC $\left(u_{\tau}\right)$ are required in order to keep the building states $\left(x_{\tau}\right)$ - like the air temperature inside the 
room - in comfortable for the users levels for all the time-steps $\tau$; moreover, the transition to the desired states should be achieved using as less energy as possible, thus operating the AC system (or equivalently selecting the $u_{\tau}$ ) intelligently. The cost function (4) is a (usually non-linear) function defining on one hand the user comfort levels and forcing the system states to that direction, and on the other hand penalizing solutions that excessively use the AC system, thus consuming a substantial amount of energy.

The proper formulation of the cost function $J$ is crucial, since it contains the definition of the problem and describes the desired states of the system. A poorly designed cost function can lead the building to undesired (or even dangerous, if for example we are controlling the $\mathrm{CO}_{2}$ concentrations) states or to cost-intensive solutions. The cost-minimization part of the cost function in the building domain is not limited only in energy reduction; many other cost indexes can be used, depending on their suitability for the problem, like the maximization of the net-energy produced, the minimization of the operational cost of the building, the maximization of the renewable-energy consumption, etc.

Model Predictive Control In the load-shifting problem at hand, the stochasticity injected in the system by the weather, forces the adoption of a formulation for the problem that will take into account all random factors. The use of a static rule-based controller to tackle the problem, defining a default control strategy applied every day, can lead to poor results, since the weather variations affecting the renewable-energy production can yield that an efficient controller for one day may result in inefficient behavior on a different day. Moreover, even if a dynamic controller, altering every day is designed for a large period of time, its performance will be deteriorated due to the unavailability of accurate weather predictions for long time periods.

To overcome the disturbances originating from the weather conditions, a model-assisted control technique is developed, in accordance to Model Predictive Control (MPC) (or Receding Horizon Control (RHC)) approaches [see Goodwin et al. 2005 and Bertsekas 1995], already used successfully in buildings domain [Oldewurtel et al. 2011a]. These control methods use a model of the process, to obtain a controller that minimizes a given cost function and, at the same time, satisfies a set of constraints, for a pre-defined time window, called the prediction horizon. More formally, the problem is defined as [Goodwin et al. 2005]:

$$
\begin{gathered}
\min \sum_{\tau=0}^{T} J_{\tau}, \\
\text { s.t }\left\{\begin{array}{c}
x_{\tau+1}=f_{\tau}\left(x_{\tau}, u_{\tau}\right), \forall \tau \in\{0,1, \ldots, T\} \\
u_{\tau} \in \mathbb{U}, \forall \tau \in\{0,1, \ldots, T-1\} \\
x_{\tau} \in \mathbb{X}, \forall \tau \in\{0,1, \ldots, T\}
\end{array}\right.
\end{gathered}
$$

Again, here, $x_{\tau} \in \mathbb{X}$ are the states, $u_{\tau} \in \mathbb{U}$ are the actions; $\mathbb{X} \in \mathbb{R}^{m}$ and $\mathbb{U} \in \mathbb{R}^{n}$ are constrained sets; $T$ is the prediction horizon; $\tau \in\{0,1, \ldots, T\}$ is the time-index; and $J$ is the cost function. 

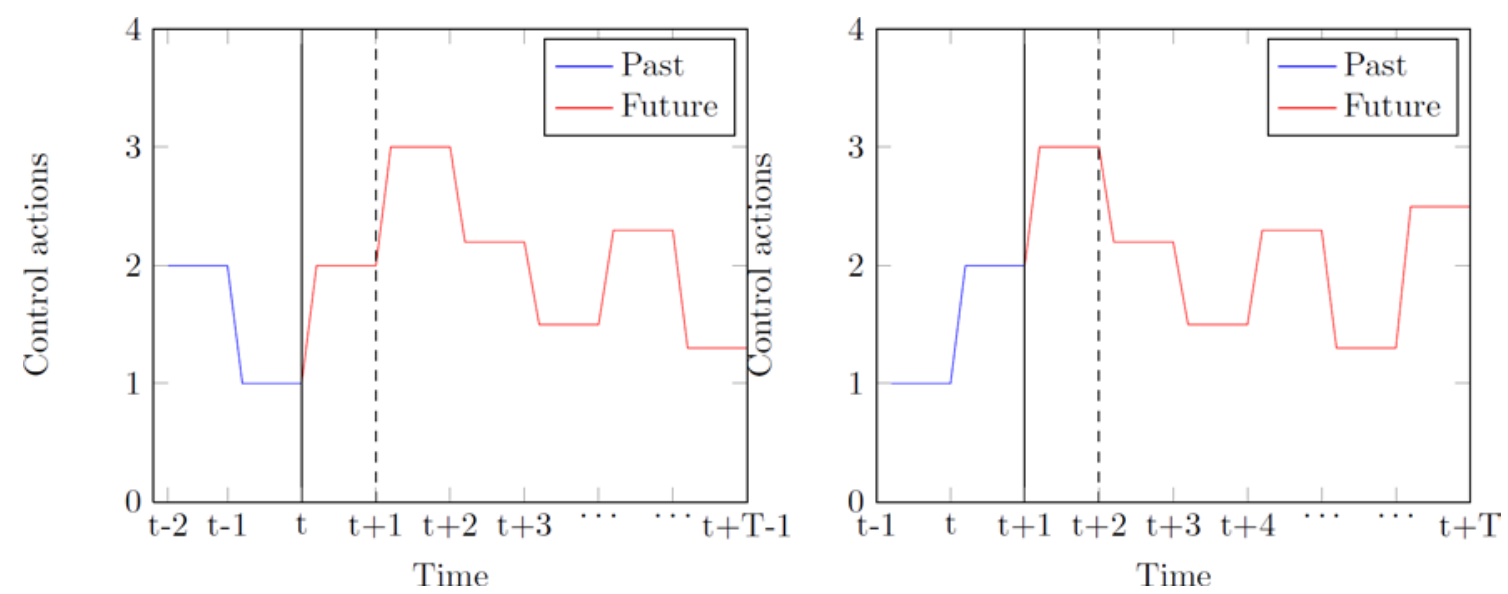

Figure 1. Model Predictive Control at time-step $\tau$ (left) and at time-step $\tau+1$ (right)

In this setting, a controller is designed based on the cost function for a period of time (prediction horizon) $T$, which is the limited time-window where accurate weather forecasts are available. Then, the controller is applied for a period of time $T^{\prime} \ll T$ (the control horizon) and a new controller is designed for the prediction horizon. A schematic representation of the process is shown in Figure 1 [Goodwin et al. 2005]:

- At time $t$ (current time), solve the control problem for the prediction horizon $[\tau, \tau+\mathrm{T}-1]$, based on the system model.

- Apply the resulting controller only for the control horizon $[\tau, \tau+1]$ and repeat for the next timestep.

Although traditional, fixed-horizon approaches can be applied to solve this problem, by defining the control horizon to coincide with the prediction horizon, they are unable to react to unpredicted disturbances acting upon the system and they tend to neglect the last time-steps of the process, since there is not enough time left for significant improvement on the cost function [Goodwin et al. 2005]. Moreover, due to their formalization they would reject newer, more accurate weather predictions.

As an example, assume that for an office building equipped with one AC system in each office, located in Athens, Greece, relatively accurate weather predictions are available for the next 24h and the forecasts are updated every three hours, for the summer period. If we design a controller based on the $24 \mathrm{~h}$ predictions and apply for the next $24 \mathrm{~h}$, the controller will not take into account the updated and more accurate weather forecasts, which could affect significantly the building behavior (e.g. an unpredicted rain alters the thermal conductivity of the walls, a cloudy sky affects the energy production of the PVs, a sudden wind change alters the behavior of the wind turbines, etc.). In addition, disturbances caused by the users of the building may occur during the day: a scheduled conference may be canceled, so cooling the conference room is no longer necessary or some users can exit the building during working hours for a significant amount of time, so the ACs to the respective offices can be switched-off to save energy.

In this example, the prediction horizon for the model-assisted controller will be selected to be 24h, determined by the weather forecasts. If we take into account only the weather disturbances, the control horizon will be $3 \mathrm{~h}$ and a new control design process will be trigger by the weather prediction updates. If we demand the controller to be able to rapidly recover from disturbances caused by user behavior, the control horizon can vary from 10min to $1 \mathrm{~h}$, depending on the speed of the control design process and the desired accuracy.

Another important consideration in the design phase of a building controller is the determination and use of a warming-up (or settling) period. The building materials store thermal energy which release in 
the future, so a different control strategy should be followed if a building is charged or not. For example, concrete buildings during winter usually pre-heat and store energy before users enter the building and progressively radiate thermal energy throughout the day. A controller designed at noon, should be aware of the thermal status of the building at the time to avoid unnecessary control actions, like reheating the building. For that reason, experimental trials are performed to determine the warming-up period (usually $T^{w}$ days) of the building and the building model is initiated from time-step $\tau-T^{w}$, using historical data, in order to provide to the control design process an accurate approximation of the thermal state of the building.

Co-Simulation It is obvious that a controller evaluation on the thermal simulation model of the building depends on the ability to perform simulations using dynamic schedules. Forecasted weather and occupancy data have to be injected in the simulated model, along with past states of the building for the warming-up phase and dynamic control inputs produced by the control strategy. In other words, the building thermal simulation model must interact dynamically with the control software. This is achieved using the co-simulation method [Nghiem et al. 2011, Privara et al. 2011, Sagerschnig et al. 2011], which consists of the use of different software components for run-time coupling.

In our case, the building model is available in EnergyPlus and the code for the control design and validation in Matlab, hence the effective utilization of the simulated model of the building is based on the establishment of a dynamic connection between EnergyPlus models and Matlab scripts implementing the control strategies. Such a connection can be achieved using EnergyPlus with External Interfaces and especially with the Building Controls Virtual Test Bed (BCVTB). The Building Controls Virtual Test Bed is a software environment, developed by Lawrence Berkeley National Laboratory which allows the coupling of different software codes for distributed simulation. The BCVTB allows simulation of the building envelope and HVAC system in EnergyPlus and implementation of the control logic in MATLAB (or other general purpose programming languages), facilitating dynamic data exchange between the two programs at each time step of the simulation [Wetter 2011]. Figure 2 [Crawley et al. 2001] shows the architecture of the connection between EnergyPlus and the BCVTB and the connection between MATLAB and BCVTB.

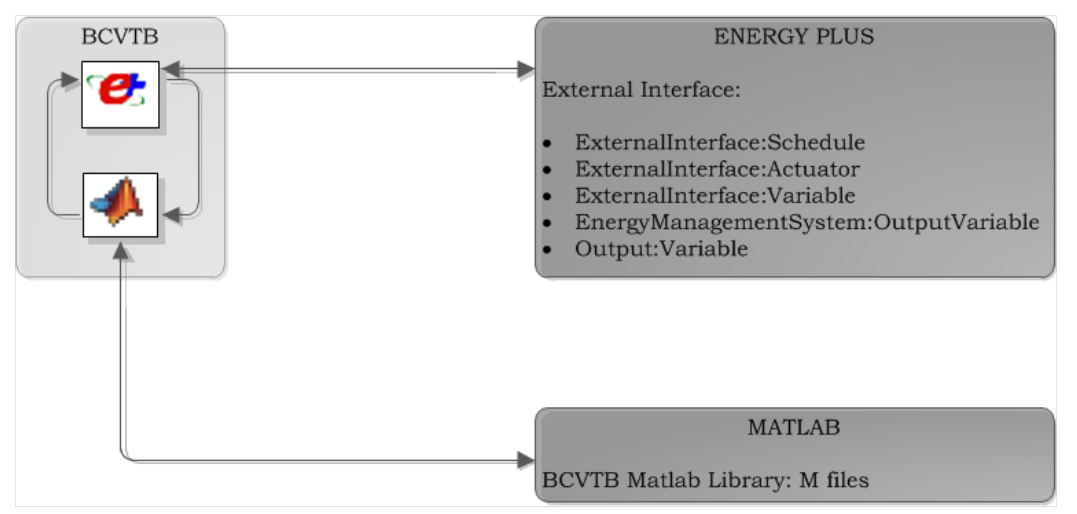

Figure 2. Ptolemy Model for exchanging data between EnergyPlus and Matlab

Cognitive-based Adaptive Optimization Algorithm with Constraints The optimization problem defined in (5) cannot be solved analytically, since it is non-linear, non-convex, an analytical state-space model is not available (since we are using the EnergyPlus simulator) and the optimal solution is difficult, if impossible, to be determined. Moreover, each controller evaluation on the model is expensive, due to the detailed nature of the building thermal simulation.

To overcome these obstacles, a fast and efficient stochastic optimization algorithm is used, called Cognitive-based Adaptive Optimization Algorithm with Constraints (CAO-C). A first application of 
the algorithm is used in traffic domain and it is called the Adaptive Fine-Tuning algorithm (AFT) [Kosmatopoulos 2009, Kouvelas et al. 2011]. There, AFT is used as an on-line algorithm, in which every day the cost function is approximated by a non-linear estimator using available data from past days, and a set of candidate, new controllers is evaluated on the estimator and not on the actual model of the system, to save simulation time. This on-line approach can lead to high convergence times and to oscillating controllers, since the divergence between the samples is very high (e.g. traffic data both from Sunday afternoons and Monday mornings, data after car-crashes, etc.). In addition, in AFT algorithm two types of approximators are used: estimators using polynomial basis functions $(S(x)=$ $\left.\alpha_{1} x^{3}+\alpha_{2} x^{2}+\alpha_{3} x+\alpha_{4}\right)$ and sigmoid basis functions $\left(S(x)=\tanh \left(\lambda_{1} x+\lambda_{2}\right)\right)$, where the initial values for the $\alpha_{i}$ and $\lambda_{i}$ are provided by the user after experimental trials on the domain of study - a laborious and time- and performance-consuming fine-tuning process. Subsequently, these values are automatically altered to minimize the approximation error of the estimator, which could lead to overfitting behavior. Finally, the candidate controllers are created by drawing random samples, using a Gaussian distribution, around the best controller so far in the process. The variance of the Gaussian in this approach has to be reduced progressively and set to zero upon convergence [Robbins et al. 1951] by hand, thus manually balancing the exploration-exploitation trade-off.

Acknowledging the aforementioned limitations, a new, offline version of the algorithm, called the Cognitive-based Adaptive Optimization (CAO) is developed and applied in energy-efficiency tasks in buildings [Giannakis et al. 2011, Kontes et al. 2011, Pichler et al. 2011], using Support Vector Machines (SVM) for the construction of the approximator [Scholkopf et al. 2002, Bishop 2006, Vapnik et al. 1996], which are widely used in classification and regression applications. For the problem at hand, v-SVR regression [Scholkopf et al. 2000], along with cross-validation [Kohavi 1995, Mosteller 1948] for the determination of the best SVM parameters [Lin et al. 2004] is used.

The necessity of an automated parameter-selection process becomes clear, when the accuracy and prediction properties of the different estimators of Figure 3 are analyzed. The real data are plotted with blue circles, and for various values of the free parameters, the different estimators are plotted. It is obvious that without model selection $(\gamma=0.0156, \mathrm{C}=1, v=0.7)$ it is practically infeasible to finetune the free parameters of the SVM estimator (and any estimator) by hand, especially when the data "live" in high-dimensional spaces. Moreover, since the shape of the data changes over time as the optimization process evolves, the best set of parameters must be adapted in each time step - a process hard to fine-tune beforehand. In addition, SVM regressors require the final approximator to be as smooth as possible, thus avoiding overfitting hazards.

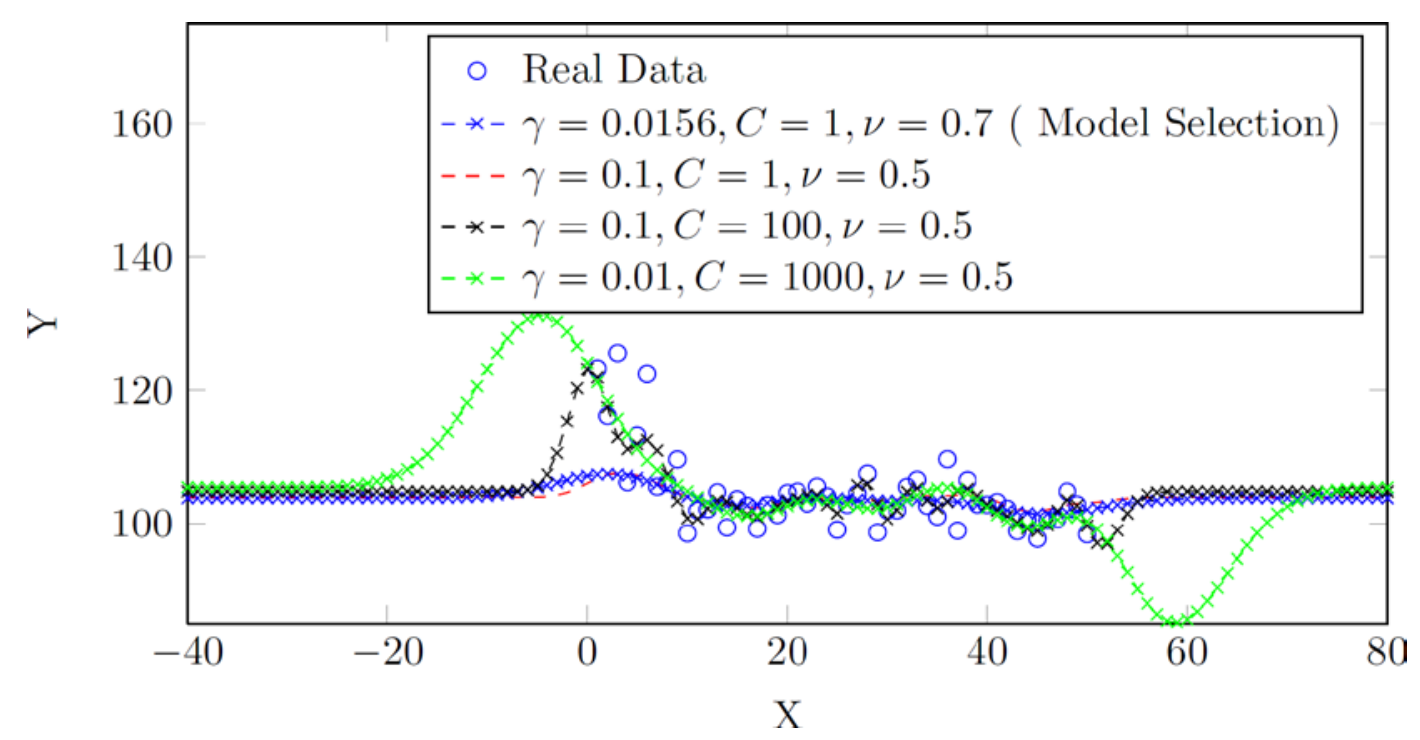

Figure 3. Estimators for different values of $C, \gamma$ and $v$ 
Another appealing property of SVMs is their prediction capability. Most estimators (including polynomial and sigmoid-based) fail to provide robust results, due to their behavior outside their "confidence interval," i.e. the limited space where data exist, leading to inaccurate results. SVM regressors on the other hand, approximate the area outside data samples with an unbiased flat line (Figure 3), thus improving the prediction accuracy of the estimator.

Finally, practical applications of SVM approximators [Giannakis et al. 2011, Pichler et al. 2011, Kontes et al. 2011] indicate that the use of laborious and, often times ambiguous, selection of RobbinsMunroe sequences of learning rates [Robbins et al. 1951] for the construction of the candidate controllers is not required for convergence.

Althoug CAO algorithm has proven fast, relieable and efficient, it incorporates user comfort constraints as penalizing terms in the cost function. This requires some additional effort to tune the trade-off between energy consumption and user comfort [Kontes et al. 2011], and this tuning becomes less intuitive when the performance index of the cost function is more complex, like in the load shifting application at hand, or when the size of the building increases and there are many comfort constraints involved. For that reason, an extension of CAO algorithm is presented here, the Cognitive-based Adaptive Optimization Algorithm with Constraints (CAO-C), where the optimization problem in (5) is transformed in:

$$
\begin{gathered}
\min \sum_{\tau=0}^{T} J_{\tau}\left(u_{\tau}\right), \\
\text { s.t }\left\{\begin{array}{c}
x_{\tau+1}=f_{\tau}\left(x_{\tau}, u_{\tau}\right), \forall \tau \in\{0,1, \ldots, T\} \\
u_{\tau} \in \mathbb{U}, \forall \tau \in\{0,1, \ldots, T-1\} \\
C\left(x_{\tau}\right)<0, \forall \tau \in\{0,1, \ldots, T\}
\end{array}\right.
\end{gathered}
$$

Here, the cost function $J$ includes only the performance index (e.g. the energy consumption), and a vector function $C\left(x_{\tau}\right)$ defining the comfort constraints is used to avoid hand-tuning the penalty terms for the user comfort in the cost function. Since the performance index (like energy consumption, total cost, etc.) depends only on the actuating components of the building (e.g. the air-conditioning system or the shading system), only the input vector is included in the cost function. In general though, the cost function is defined by the nature of the problem and can include any combination of states and actions.

In this approach, first, a Support Vector Machine (SVM) estimator $\hat{J}$ for the cost function is constructed:

$$
\hat{J}_{k(\theta)}=\vartheta_{k}^{T} \phi(\theta)
$$

Here, $\hat{J}$ is the approximation/estimation of $J$ generated at the $k$-th iteration of the algorithm; $\phi$ denotes the nonlinear vector of $L$ regressor terms; $\theta$ is the controller; and $\vartheta_{k} \in \mathbb{R}^{L}$ denotes the vector of parameter estimates calculated at the $k$-th iteration. Practically, this equation (in one dimension) defines a regression function as in Figure 3, where the controllers $\theta$ are in the x-axis and the respective score on the performance index is in y-axis.

Subsequently, one SVM approximator for each constraint in the vector function $C(x)$ is defined:

$$
\widehat{\mathrm{C}}_{\mathrm{k}}(\theta)=\vartheta_{\mathrm{k}}^{(\mathrm{C}) \mathrm{T}} \phi^{(C)}(\theta)
$$


Here $\hat{C}_{k}$ denotes the estimation of the constraint function $C$ generated at the $k$-th iteration, $\phi^{(C)}$ denotes the nonlinear vector of $L_{1}$ regressor terms, $\theta$ is the controller, $\vartheta_{\mathrm{k}}^{(\mathrm{C})} \in \mathbb{R}^{L_{1}}$ denotes the vector of parameter estimates calculated at the $k$-th iteration and $L_{1}$ is a positive integer denoting the size of the function approximator. In general, one approximator (as in Figure 3 for one dimension) for each constraint function is constructed, in which x-axis contains the controllers $\theta$ and $y$-axis the respective constraint value.

As soon as the estimators $\hat{J}_{k}$ and $\hat{C}_{k}$ are constructed, the best controller $\bar{\theta}$ so far in the optimization process is selected, and a set $\Theta_{c}$ of candidate controllers around the best one is created:

$$
\Theta_{c}=\left\{\theta^{(j)} \mid \bar{\theta}+\alpha \zeta_{k}^{(j)}, j \in\{0, \ldots, N\}, \zeta_{k}^{(j)} \sim \mathcal{N}(0,1)\right\}
$$

In (9), $\zeta_{\mathrm{k}}^{(\mathrm{j})}$ is a zero-mean, unity-variance random vector with dimension equal to the dimension of $\bar{\theta}$ and $\alpha$ is a vector of the same size, used as an exploration term in the control parameter space. As stated before, $\alpha$ remains the same throughout the optimization process, due to the use of SVMs.

Subsequently, each candidate (random) controller created by (9) is evaluated on the cost function and constraint estimators. For example, in one dimension (Figure 3), having the estimators at hand (regression line) allows to predict the cost and constraint values of random controllers $\theta^{(j)}$ by projection on the regression line. After all candidate controllers have been evaluated, the controller that satisfies all comfort constraints (as predicted by the estimators $\hat{C}_{k}$ ) and has the lowest (for minimization) or the highest (for maximization) score (as predicted by the approximation $\hat{J}_{k}$ of the performance index) is selected to be tested on the simulator for the next iteration of the algorithm. If all candidate controllers do not satisfy all the constraints, the controller which less violates the constraints is selected for the next iteration. Note here, that estimating the performance of a controller on the approximator is computationally much cheaper than evaluating on the simulator. The whole process is repeated until the algorithm converges to a final controller.

Methodology Connecting all the necessary parts analyzed in this section, the general methodology followed emerges: given weather and occupancy predictions for a building in a specific geographical area, and assuming a detailed building thermal simulation model is available, CAO-C algorithm is initiated in predefined time intervals and using a) available past data of the building for the warm-up process, b) the co-simulation approach to evaluate candidate controllers in the simulator, and c) SVM estimators for the cost function and the constraints, produces a controller applied to the physical building.

\section{EXPERIMENTS}

The Building Model To illustrate the proposed approach, a sample three-zone building, as in Giannakis et al. [2011], is considered and a detailed thermal simulation model of the building in EnergyPlus is constructed. For demonstration purposes and use of available weather data for the year 2010 from a Meteonorm [Remund et al. 1999] weather file, the building is supposed to be located in Athens, Greece and the simulation time step is set at 10 minutes. Obviously, any other location could have been chosen, which would affect the weather data used and the algorithms that use latitudinal and longitudinal information for calculation of solar paths. 

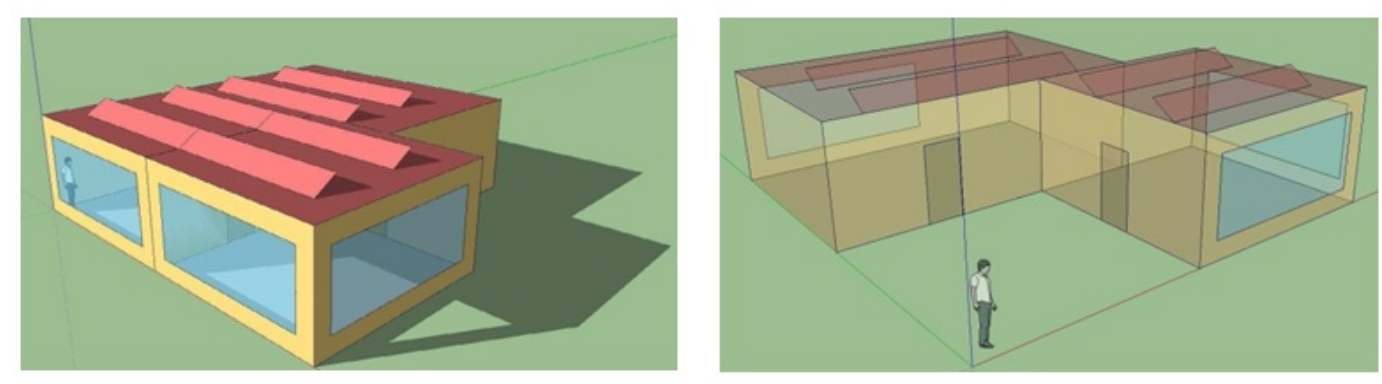

Figure 4. The test building

A detailed representation of the building geometry is shown in Figure 4, created using the OpenStudio plugin for Google SketchUp. The office building comprises 3 office rooms and since split type air conditioners are installed on each room, the temperature can be independently adjusted, thus each room of the building is defined as a separate thermal zone. EnergyPlus uses the characteristics of the construction materials in order to calculate the heat transfer through the walls, floor, roof and glazing. In general, the building's envelope insulation is insufficient. The U-values are quite high which reveals the thermal attitude of the building. The building has a significant amount of thermal losses due to its construction materials and solar gains during summer due to its large glazing area. The various components of the building, along with each layer's construction are described in detail in Kontes et al. [2011].

In addition, six photovoltaic arrays are installed, each of which consists of four photovoltaic panels, totaling $6 \mathrm{KW}$ nominal power, and entirely cover the corresponding sloping surface that is attached to the roof. To define internal gains due to occupant presence, data for the number of occupants on each zone, occupancy timetables and metabolic rates (108 W/Person) for office activities are imported to the thermal model. The working days are Monday till Friday except from Christmas break, Easter break, summer break and some local holidays, and the building is occupied from 6:00 a.m. to 6:00 p.m. during working days.

Heat gains due to infiltration and ventilation can be significant and for this reason a detailed modeling of the infiltration is performed using EnergyPlus AirflowNetwork. Infiltration is the unintended flow of air from the outdoor environment directly into a thermal zone. Infiltration is generally caused by the opening and closing of exterior doors, cracks around windows, and even in very small amounts through building elements [Crawley et al. 2001]. Ventilation is the purposeful flow of air from the outdoor environment directly into a thermal zone in order to provide some amount of non-mechanical cooling. The AirflowNetwork model provides the ability to calculate multi-zone airflows driven by outdoor wind and forced air during AC system operation [Crawley et al. 2001].

With respect to Heating Ventilation and Air Conditioning (HVAC) system of the building, heating is achieved through a central system using an oil boiler and hot water radiators in each room. A packaged terminal heat pump is positioned in each office to provide cooling during summer months, but can also used as a supplementary heating system whenever the central system does not cover the heating needs of each space during winter. To model the HVAC system in EnergyPlus, a dual setpoint thermostat is introduced for each of the three zones with separate heating and cooling setpoint temperatures. Data regarding the heating and cooling function of each type of split unit in each zone, such as availability schedule, air flow rates, EER, COP and capacity are introduced. An extensive and analytical presentation of each component of the building is available in Kontes et al. [2011].

User Comfort Mathematical models, to estimate thermal sensation of people in their environment based on personal, environmental and physiological variables that influence thermal comfort has been the subject of many studies. The most widely known model has been developed by Fanger [ASHRAE 2004]. According to that, the Predicted Mean Vote (PMV) index predicts the mean response of a large 
group of people according to the ASHRAE 7-point thermal sensation scale. The Predicted Percent of Dissatisfied people (PPD) is an index which quantifies the thermal comfort of a group of people at a particular thermal environment. EnergyPlus calculates PPD and PMV indices at each timestep of the simulation.

Plenty of methodologies for measuring thermal comfort exist [ASHRAE 2009]. While many are accepted as accurate, each relies on a different set of assumptions. Every acceptable measure of comfort could be selected, but in the present work we choose the Fanger PMV (or equivalently the PPD) index, as it appears on many European standards. Note here that these metrics are not equivalent when it comes to the optimization problem, so they can influence the control design process and lead to different resulting controllers.

Setup of the Optimization Problem Assuming that a day-ahead notification on a (hypothetical) tariff plan is provided, the Building Energy Management System — taking into account available weather forecasts and occupancy schedule predictions - is requested to design a control strategy that keeps the users comfortable (with respect to the Fanger PPD comfort index) and exploits the power produced by the PVs to save energy and minimize the cost of the electric bill for a hot summer day (June 6, 2010), using only the AC system available in each zone. We assume that there is no energy storage and selling-back to the grid is not allowed. Moreover, a hypothetical tariff is used, by defining the power used from the grid after 11:30 a.m. to be three times more expensive, forcing the algorithm to exploit the power produced by the PV.

Assuming the predefined building schedule (occupancy between 06:00 and 18:00), the controller is defined as $\theta \in \mathbb{R}^{3 \times 13}$, applying a one-hour set-point temperature for each zone's AC for the time each zone is occupied. The set-points are integers in $[22,30]$. More analytically, the controller is (Table 1):

Table 1

The controller applied

\begin{tabular}{c|c|c|c|c}
\multirow{2}{*}{} & \multicolumn{4}{|c}{ Setpoints } \\
\cline { 2 - 5 } & $\begin{array}{c}\text { Night } \\
\mathbf{0 0 : 0 0 - 0 5 : 0 0}\end{array}$ & $\begin{array}{c}\text { Pre-cooling } \\
\mathbf{0 5 : 0 0 - 0 6 : 0 0}\end{array}$ & $\begin{array}{c}\text { Occupancy } \\
\mathbf{0 6 : 0 0 - 1 8 : 0 0}\end{array}$ & $\begin{array}{c}\text { Night } \\
\mathbf{1 8 : 0 0}-\mathbf{0 0 : 0 0}\end{array}$ \\
\hline West Zone & $30^{\circ} \mathrm{C}$ & $\theta_{1,1}$ & $\theta_{1,2}-\theta_{1,13}$ & $30^{\circ} \mathrm{C}$ \\
\hline East Zone & $30^{\circ} \mathrm{C}$ & $\theta_{2,1}$ & $\theta_{2,2}-\theta_{2,13}$ & $30^{\circ} \mathrm{C}$ \\
\hline North Zone & $30^{\circ} \mathrm{C}$ & $\theta_{3,1}$ & $\theta_{3,2}-\theta_{3,13}$ & $30^{\circ} \mathrm{C}$
\end{tabular}

Here, the AC systems are switched-off manually during the night (setpoint set to $30^{\circ} \mathrm{C}$ ), an hour before the users enter the building (05:00 - 06:00) is available for pre-cooling (if necessary), and each hour during the occupied interval a different setpoint is applied.

The optimization problem is defined as:

$$
\begin{gathered}
\min \frac{1}{T} \sum_{\tau=1}^{T}\left(c_{\tau} P_{\tau}^{c}-P_{\tau}^{p}\right) \text {, with } P_{\tau}^{c}-P_{\tau}^{p}=0 \text {, if } P_{\tau}^{c}-P_{\tau}^{p}<0, \\
\text { s.t. } \frac{1}{T} \sum_{\tau=1}^{T} F_{i \tau}<10, \quad \forall i \in\{1,2,3\}
\end{gathered}
$$

Here $\tau$ is the time index, with $\tau=1$ corresponding to 5 a.m. and $\tau=T$ at 6 p.m.; $P_{\tau}^{c}$ is the total power required from all the ACs of the building in each time-step $\tau$; $P_{\tau}^{p}$ is the power produced by the PV in each time-step $\tau$; $c_{\tau}$ is the tariff of the grid energy used; and $F_{i \tau}$ is the Fanger PPD index in each zone $i$ 
for time-step $\tau$. The equation $P_{\tau}^{c}-P_{\tau}^{p}=0$, if $P_{\tau}^{c}-P_{\tau}^{p}<0$ ensures that the excess power produced by the PV is not stored.

The optimization problem posed in (10) originates from the general definition in (6); the cost function $J_{\tau}=c_{\tau} P_{\tau}^{c}-P_{\tau}^{p}$ is defined in order to minimize the total energy cost and the constraint functions $C(x)=\frac{1}{T} \sum_{\tau=1}^{T} F_{i \tau}-10<0$, require the average Fanger PPD values in each zone to be below $10 \%$. The definition of the cost function fully describes the problem and enables the CAO-C algorithm to move to proper solutions: experience suggests that the best strategy for the controller in this situation would be to pre-cool the building when free-energy from the renewables is available or at least when the grid-provided energy is cheaper, and release the cooling load stored in the building later during the day; the selected cost function, by penalizing the operational cost, forces the algorithm towards discovering the best control strategy.

Results For demonstration purposes and simplicity, the prediction horizon and the control horizon in the specific test-case are defined to be the same and equal to 24h. This implies that the weather conditions and the occupancy behavior are deterministic and known in advance for each day, so the optimization process is initiated during the night and produces a controller for the following day.

First, a rule-based controller is defined for the building, keeping a constant set-point for all the zones, during the time-period the building is occupied. The total energy consumption and comfort levels for different set-points are shown on Table 2, where it is obvious that $26^{\circ} \mathrm{C}$ is the best rulebased strategy.

Table 2

Rule-based controller in Athens, June 62010

\begin{tabular}{c|c|c|c|c}
\multirow{2}{*}{$\begin{array}{c}\text { Setpoints } \\
\mathbf{T}_{\text {HVAC }}\left({ }^{\circ} \mathbf{C}\right)\end{array}$} & \multicolumn{3}{|c|}{ Fanger PPD } & $\begin{array}{c}\text { Energy } \\
\text { Consumption }\end{array}$ \\
\cline { 2 - 5 } & West Zone & East Zone & North Zone & $7.2150 \mathrm{KWh}$ \\
\hline $\mathbf{2 5 ^ { \circ } \mathbf { C }}$ & 8.2 & 7.4 & 8.3 & $4.6095 \mathrm{KWh}$ \\
\hline $\mathbf{2 6 ^ { \circ } \mathbf { C }}$ & 8.9 & 8.7 & 9.3 & $2.3342 \mathrm{KWh}$
\end{tabular}

A first experiment is initiated, applying the rule-based controller and assuming there is no PV system available. Figure 5 shows the rule-based control strategy and its affect to the West Zone of the building. Here, the AC system begins to operate after 12 p.m., and until the occupants exit the building. Fanger PPD index is below 15\% the whole time (except from 6 a.m. to 7 a.m., but at that time, an - unavailable - heating load is necessary to maintain acceptable comfort levels), which is a reasonable upper bound for this type of buildings [ASHRAE 2004]. 


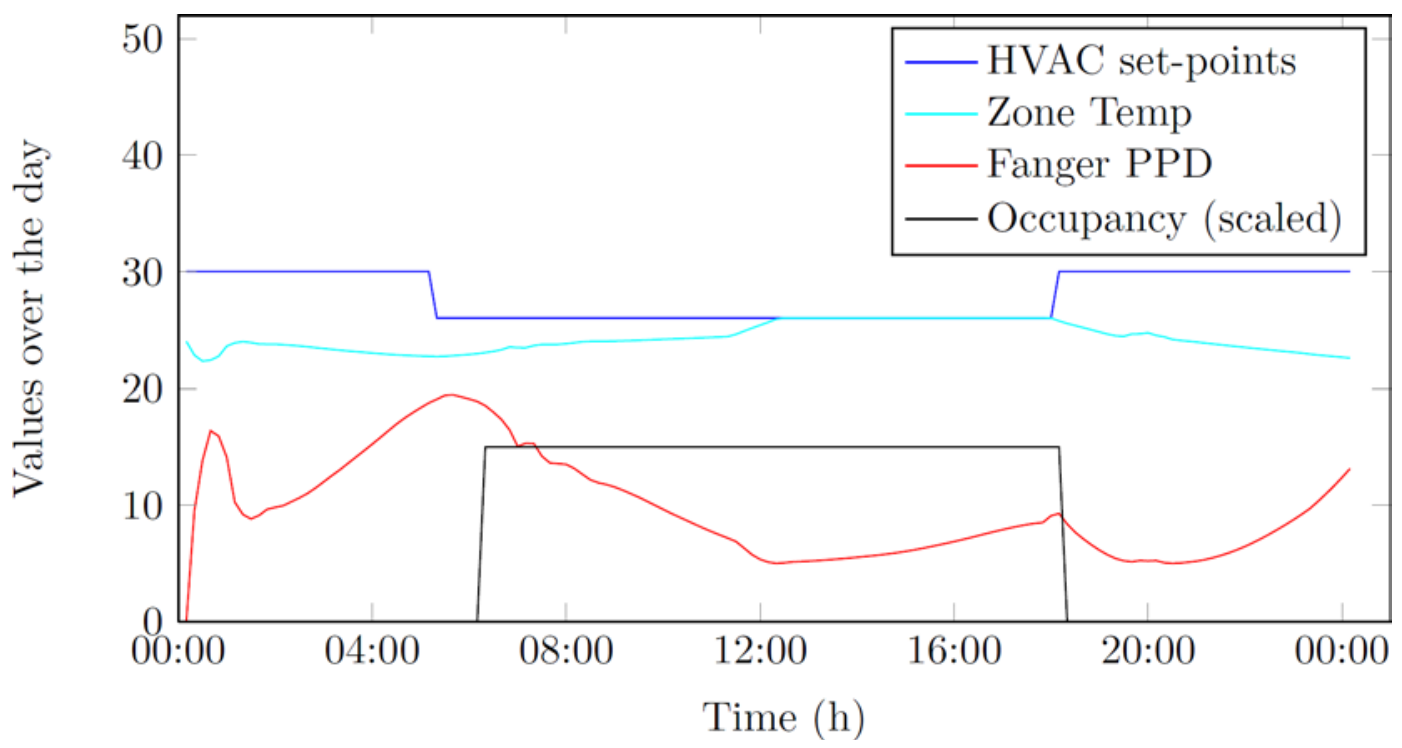

Figure 5. Rule-based control effects on West Zone, for June 6, 2010

Subsequently, an optimization process, using the CAO-C algorithm is initiated for the same day and for the same building without the PV system and the power demands for the rule-based controller and the controller designed by CAO-C are in Figure 6. The resulting controller attempts to "shift" the demand to the low-tariff area and minimize the total operational cost, by pre-cooling the zone before Fanger PPD index rises.

After this first experiment, three separate optimization processes are initiated and in each one we assume that the building is equipped with different nominal power PV systems: $6 \mathrm{KW}, 3 \mathrm{KW}$ and 2 $\mathrm{KW}$ respectively. In each case the controller has to produce control strategies that exploit the free energy produced by the PV systems.

Figure 7 shows the power demand for the rule-based controller and for the controller resulting from CAO-C, for the $6 \mathrm{KW}$ PV system. CAO-C manages to produce a controller which fully exploits the PV system, since all the power needed for the AC operation is covered by the free power produced from the PV. The cooling load is shifted using a pre-cooing strategy and is completely "fitted" under the PV production curve. The cost for the controller produced by CAO-C for this setting is 0 .

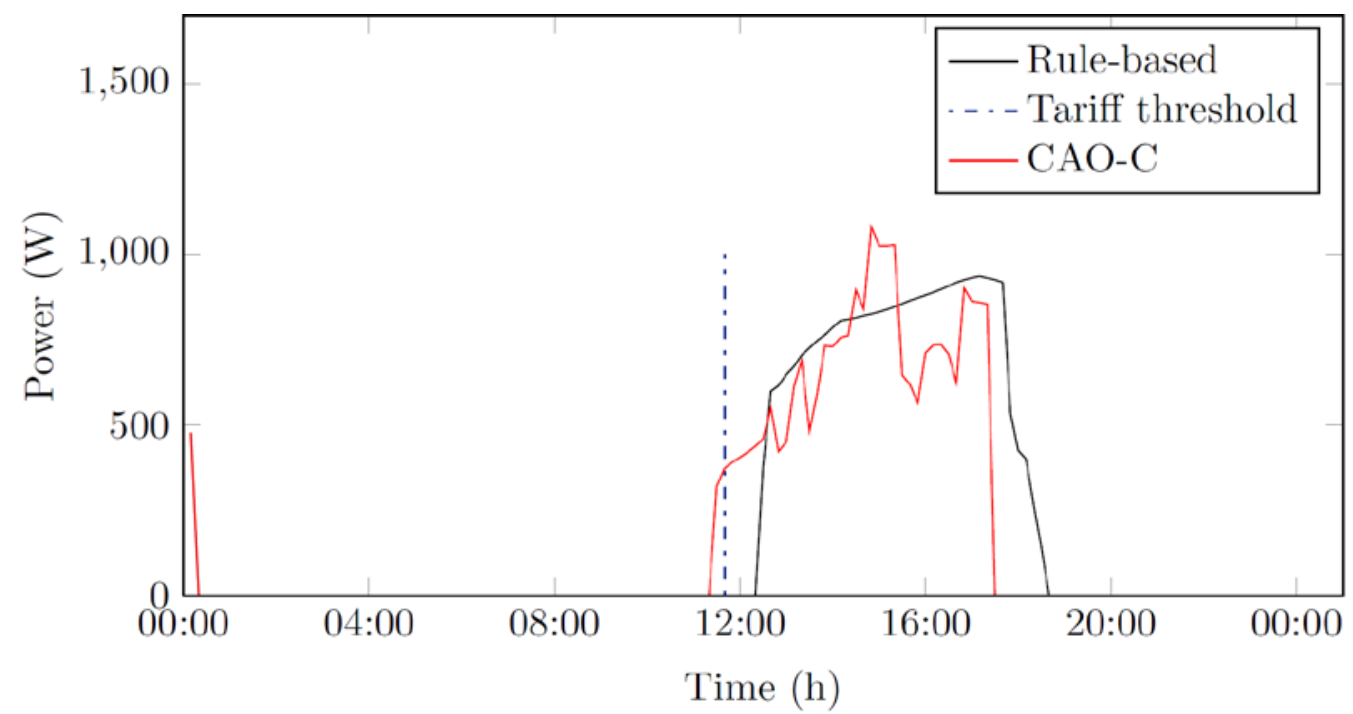

Figure 6. Power demand for rule-based controller and CAO-C controller, for June 6, 2010 
The rule-based controller on the other hand, following the static, predefined strategy is unable to adapt to the PV system presence and requires a small portion of demand not covered by the PV, thus elevating the cost due to the increased tariff (Table 3).

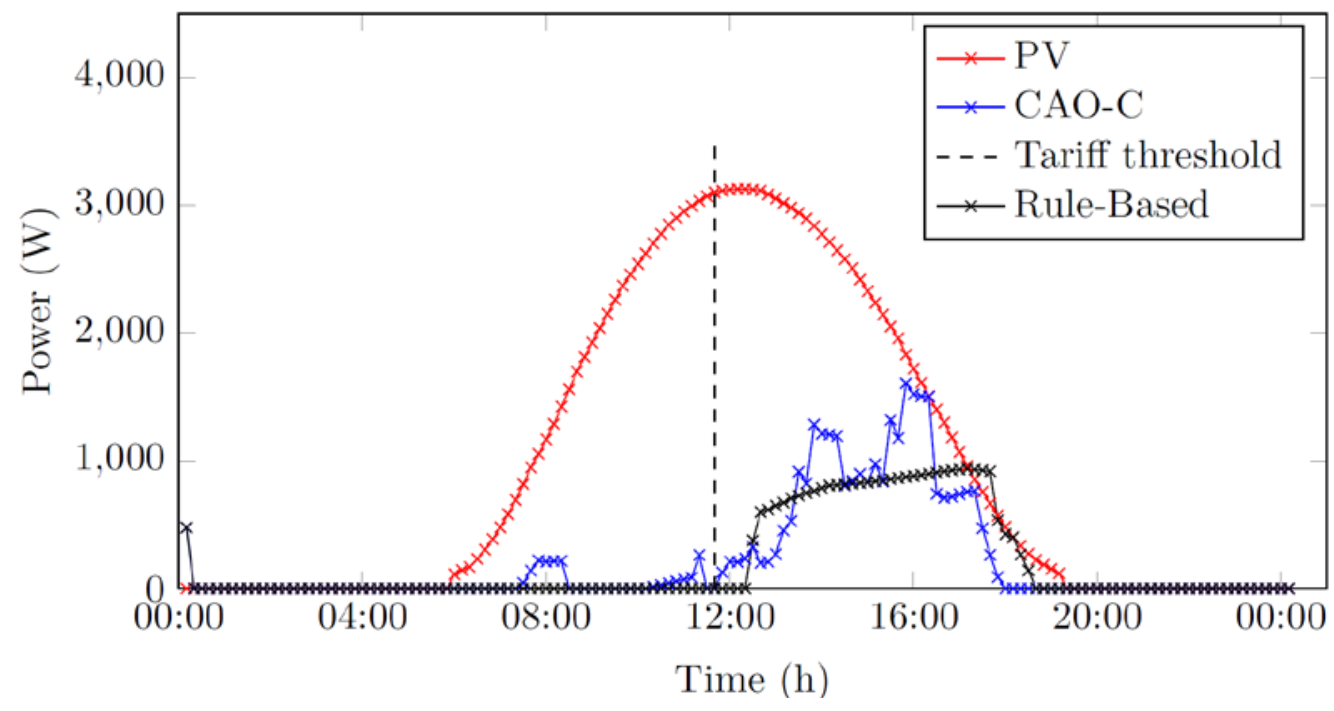

Figure 7. Power demand for rule-based controller and CAO-C controller, for June 6, 2010, assuming a $6 \mathrm{KW}$ PV system

Following, the same experiment is initiated, but with half nominal power for the PV (3 KW). Here, although the setting is different, rule-based controller follows its pre-defined control strategy, leading to significant energy use from the grid, while CAO-C on the other hand, produces a control scheme attempting to use as much power produced as possible (Figure 8), and finally achieves using the hypothetical tariffs defined - 81\% less cost (Table 3). CAO-C adapts to the reduced PV system and attempts to reshape the demand in a way to maximize the energy covered by the renewables production. A solution that would cover all the energy consumption by the specific reduced PV would require less use of the AC system and would lead to constraint violations. Note here that the high cost reduction is due to the hypothetical tariff scheme selected, in which energy consumption from the grid is heavily penalized. Nevertheless, it is an indicator of the efficiency of the algorithm. 


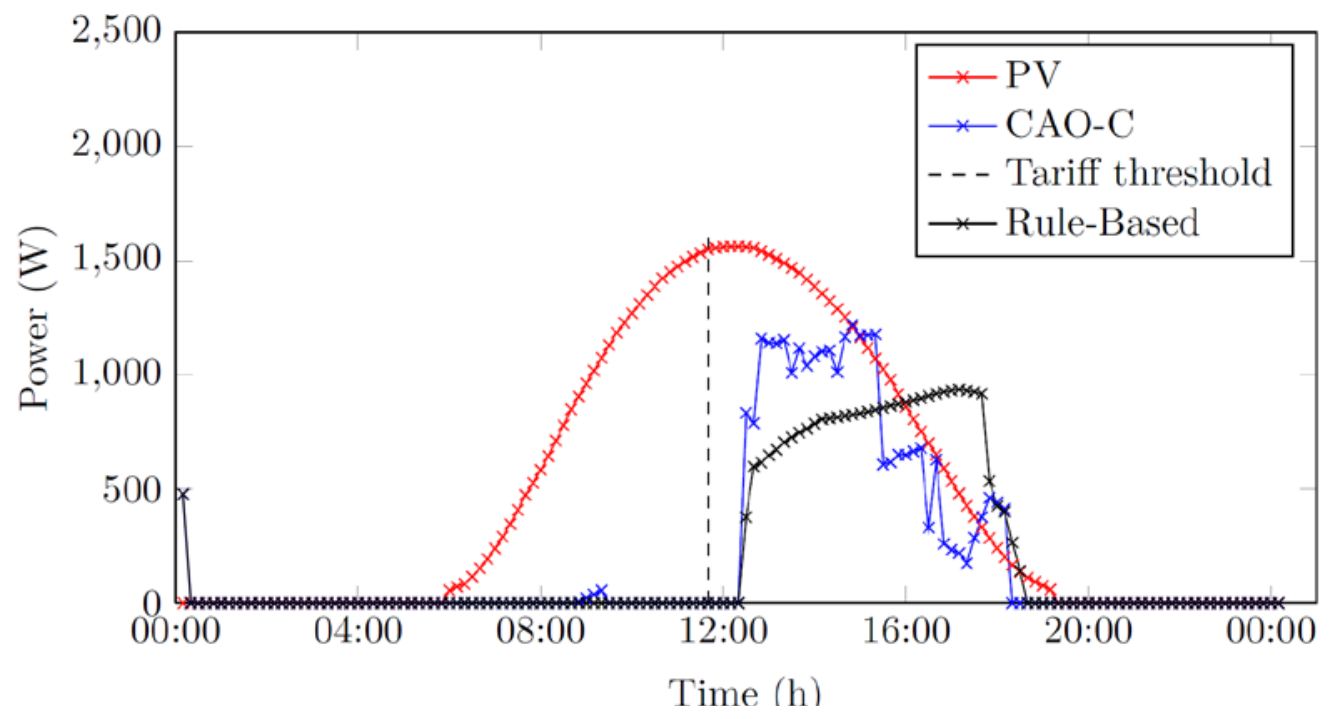

Figure 8. Power demand for rule-based controller and CAO-C controller, for June 6, 2010, assuming a $3 \mathrm{KW}$ PV system

Finally, an inefficient - with respect to the building's needs - PV system with 2 KW nominal power is used and the behavior of the resulting controller is in Figure 9. Here, once again, the rulebased controller follows the fixed strategy, unable to incorporate the energy produced by the PV system. CAO-C on the other hand attempts to shift the load under the PV production curve and results in $69.1 \%$ cost reduction (Table 3). Due to the insufficient PV system, a small amount of energy from the grid is required during the pre-cooling phase. Again, the resulting high value for the cost reduction is due to the special structure of the hypothetical tariff used, but still reflects the superiority of the solution provided by the algorithm.

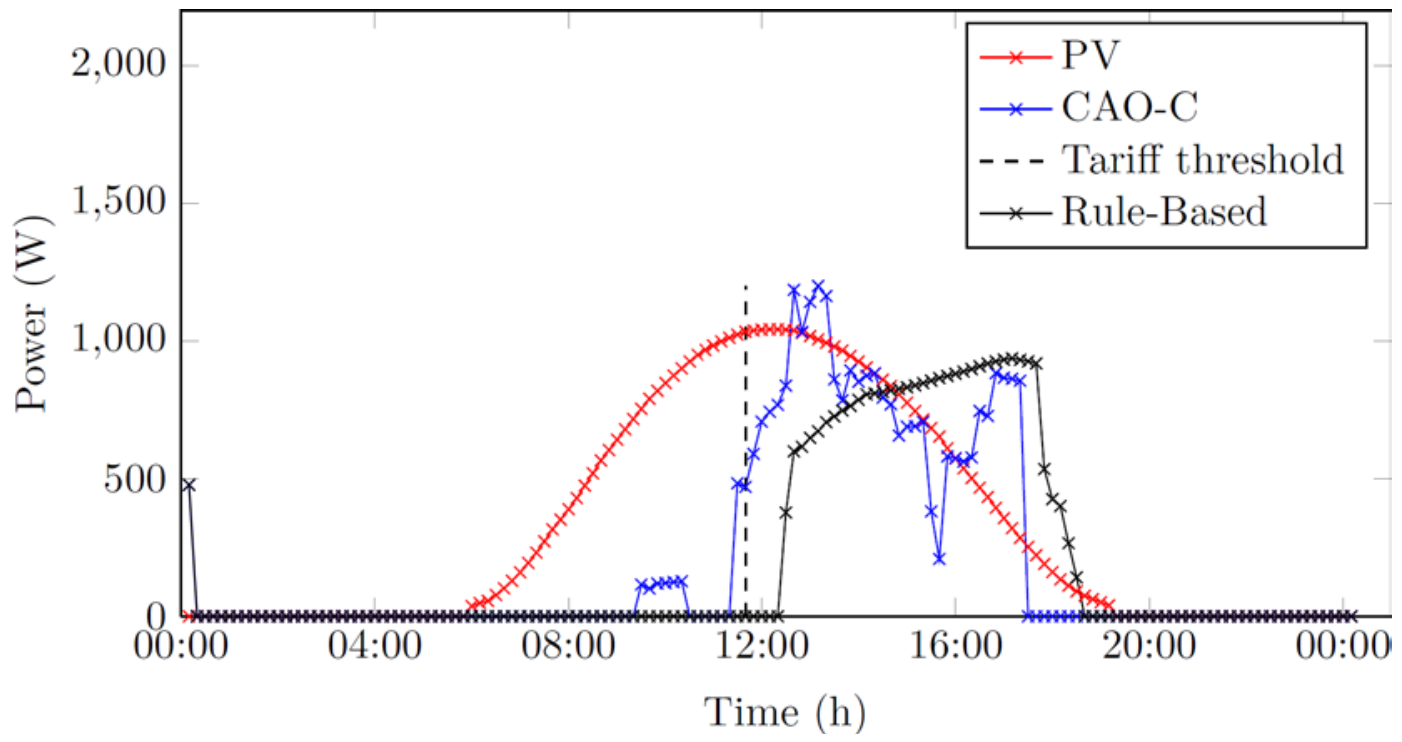

Figure 9. Power demand for rule-based controller and CAO-C controller, for June 6, 2010, assuming a $2 \mathrm{KW}$ PV system

Figure 10 shows the control strategy CAO-C algorithm concluded, for the $2 \mathrm{KW}$ PV system and for West Zone. From 10 a.m. to 12 p.m. the zone is pre-cooled, and after 12 p.m. and until occupants exit the building, the controller constantly alters the set-points, balancing the trade-off between operational cost and user comfort. 


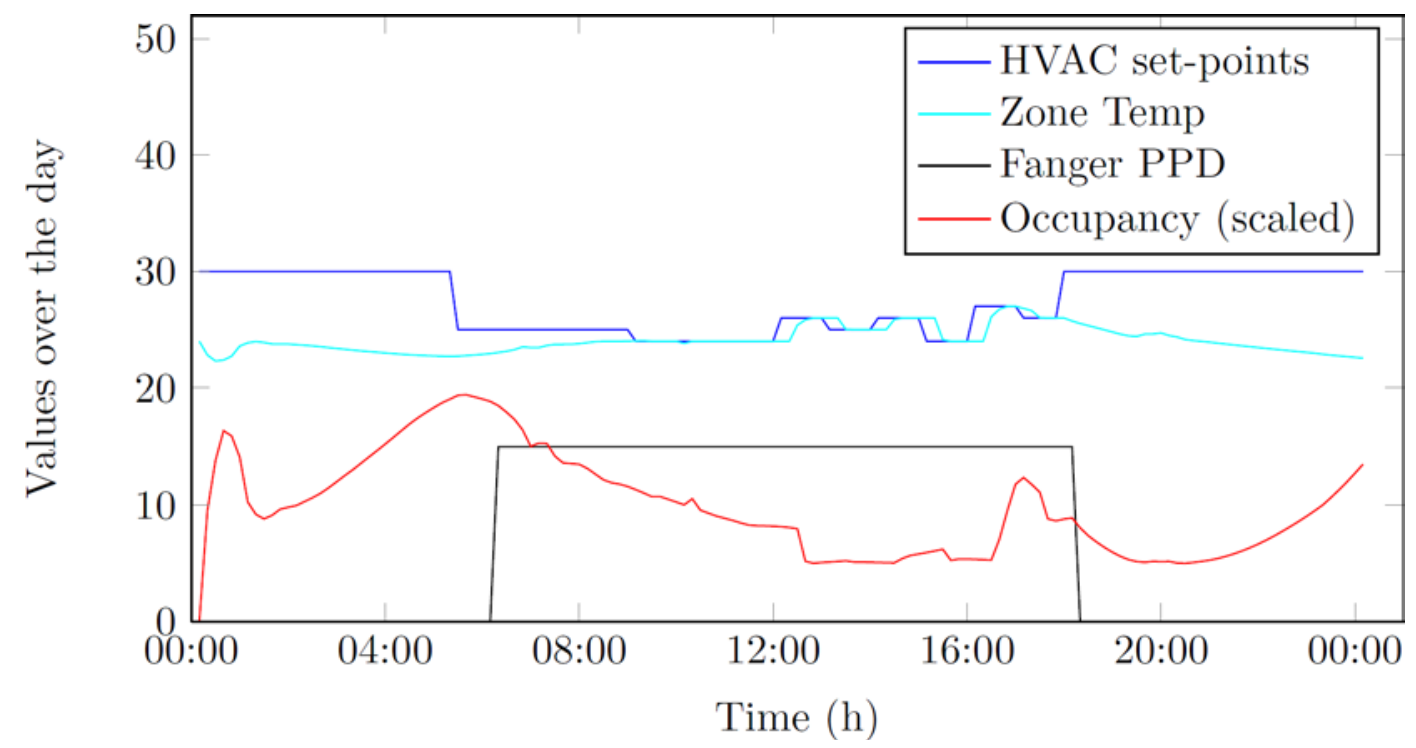

Figure 10. Control actions and effects on West Zone, for June 6, 2010, for the controller produced by CAO-C

Note here, that according to the results presented in Table 3 the controllers produced by CAO-C in two of the experiments consume more energy (in total) compared to the rule-based controller, but this energy is mostly covered by the PV. This means that under a poorly-selected (for the specific task) cost function, which would require minimization of the total energy consumption - even if the energy was produced from renewables - , the rule-based controller would be evaluated as better than the controller produced by CAO-C. Obviously, such a cost function would fail to define the problem properly and would lead to poor results.

Table 3

Results for different PV systems

\begin{tabular}{|c|c|c|c|c|c|c|c|}
\hline \multirow{2}{*}{\multicolumn{2}{|c|}{$\begin{array}{c}\text { Test Day: } \\
\text { June 6, } 2010\end{array}$}} & \multicolumn{2}{|c|}{$6 \mathrm{KW}$ PV } & \multicolumn{2}{|c|}{$3 \mathrm{KW}$ PV } & \multicolumn{2}{|c|}{$2 \mathrm{KW}$ PV } \\
\hline & & RB & CAO-C & $\mathbf{R B}$ & CAO-C & RB & CAO-C \\
\hline \multicolumn{2}{|c|}{$\begin{array}{c}\text { Cost Function } \\
\text { Value }\end{array}$} & $\begin{array}{c}1.4961 \mathrm{x} \\
10^{3}\end{array}$ & 0 & $\begin{array}{c}1.2856 \mathrm{x} \\
10^{4}\end{array}$ & $\begin{array}{c}2.4456 \mathrm{x} \\
10^{3}\end{array}$ & $\begin{array}{c}3.3243 \mathrm{x} \\
10^{4}\end{array}$ & $\begin{array}{c}1.0274 \mathrm{x} \\
10^{4}\end{array}$ \\
\hline \multicolumn{2}{|c|}{$\begin{array}{c}\text { Energy } \\
\text { Consumption }\end{array}$} & 4.6095 & 5.3925 & 4.6095 & 4.5685 & 4.6095 & 4.7170 \\
\hline \multirow{3}{*}{$\begin{array}{c}\text { Mean } \\
\text { Fanger } \\
\text { PPD } \\
(\%)\end{array}$} & $\begin{array}{l}\text { West } \\
\text { Zone }\end{array}$ & 8.9 & 9.6 & 8.9 & 9.2 & 8.9 & 9.9 \\
\hline & $\begin{array}{l}\text { East } \\
\text { Zone }\end{array}$ & 8.7 & 8.5 & 8.7 & 8.9 & 8.7 & 9.7 \\
\hline & $\begin{array}{l}\text { North } \\
\text { Zone }\end{array}$ & 9.3 & 9.1 & 9.3 & 9.9 & 9.3 & 9.6 \\
\hline
\end{tabular}

Discussion In all the experiments, $\mathrm{CAO}-\mathrm{C}$ algorithm exhibits superior behavior compared to the pre-defined, static rule-based controller. This is due to a) the ability of CAO-C to vary the set-points during the day, balancing efficiently the discomfort levels with the cost, and b) the static nature of the rule-based controller, which is unable to identify and incorporate information on the varying output profile of the renewables. CAO-C algorithm produces controllers that efficiently balance the trade-off between cost reduction and user comfort, managing to shift the cooling load of the building into the free-energy production time-window. 


\section{CONCLUSIONS}

A model-assisted methodology, along with CAO-C stochastic optimization algorithm and weather and occupancy predictions is proposed in order to assist the smoother incorporation of smart buildings into the smart grid environment. In a test building, equipped with a dedicated PV system, and for a summer day, the algorithm automatically produces control strategies that manage to significantly reduce the cost — using a hypothetical energy tariff scheme —, compared to a static, rule-based controller, by shifting the load of the building towards the time-interval in which free energy from the renewables is available. The proposed approach is a method of automatically designing Building Energy Management Systems that are able to adapt to the stochastic nature of the renewable energy sources available, caused by the microclimatic conditions of each area, and to signals from the grid. The results presented here correspond to a simple test-case, but are able to demonstrate the potential of the method. Future work, including experiments with more realistic tariffs, combinations of renewable energy sources and application in smart neighborhoods is conducted within PEBBLE FP7 project [PEBBLE 2010].

\section{ACKNOWLEDGEMENTS}

The authors would like to thank Jerome Adnot, Bruno Duplessis and Charles Fremond for the fruitful discussions and collaboration. The research leading to these results has been partially funded by the European Commission FP7-ICT-2007-9.6.3, Energy Efficiency under the contract \#248537 (PEBBLE).

\section{REFERENCES}

ASHRAE [2004], ANSI/ASHRAE Standard 55-2004: thermal environmental conditions for human occupancy. American Society of Heating, Refrigerating and Air-Conditioning Engineers, 2004.

ASHRAE [2009], ASHRAE Handbook - Fundanmentals, American Society of Heating, Refrigerating and Air-Conditioning Engineers, 2009.

Azzopardi, B. and Mutale, J. [2009], Smart integration of future grid-connected PV systems, 34th IEEE Photovoltaic Specialists Conference (PVSC), 2009, 2364-2369.

Bertsekas, Dimitri P. [1995], Dynamic Programming and Optimal Control, Athena Scientific, 1995. Bishop, C.M. [2006], Pattern recognition and machine learnin,. Springer New York, 2006.

Crawley, D.B., Lawrie, L.K., Winkelmann, F.C. et al. [2001], EnergyPlus: creating a newgeneration building energy simulation program, Energy and Buildings, 2001, pp 319-331.

Gamauf, T., Leber, T., Pollhammer, K. and Kupzog, F. [2011], A generalized load management gateway coupling smart buildings to the grid, AFRICON, 2011.

Giannakis, G.I., Kontes, G.D., Kosmatopoulos, E.B and Rovas, D.V. [2011], A model-assisted adaptive controller fine-tunning methodology for efficient energy use in buildings, Mediterranean Conference on Control \& Automation. Corfu, Greece, 2011, 49-54.

Goodwin, G.C., Seron, M. and De Dona, J. [2005], Constrained control and estimation: an optimisation approach, Springer Verlag, 2005.

Haase, T., Hoh, A., Matthes, P. and Muller, D. [2007], Integrated simulation of building structure and building services installations with Modelica, Proceedings of Roomven. 2007.

Hashmi, M., Hanninen, S. and Maki, K. [2011], Survey of smart grid concepts, architectures, and technological demonstrations worldwide, 2011 IEEE PES Conference on Innovative Smart Grid Technologies (ISGT Latin America), 2011, 1-7.

International Energy Agency [2007], Task XI : Time of Use Pricing and Energy Use for Demand Management Delivery. Final Report. Report no: 6178. Demand-Side Management Programme, International Energy Agency, 2007. 
Klein, S.A., Beckman, W.A. and Duffie, J.A. [1976], TRNSYS - A TRANSIENT SIMULATION PROGRAM, ASHRAE Transactions, Vol. 82 (1976), pp 623-633.

Kohavi, R. [1995], A study of cross-validation and bootstrap for accuracy estimation and model selection, International Joint Conference on Artificial Intelligence, 1995, 1137-1145.

Kontes, G.D., Giannakis, G.I., Lilis, G.N., Kosmatopoulos, E.B. and Rovas, D.V. [2011], PEBBLE BO\&C System, PEBBLE Deliverable 3.2, 2011.

Kosmatopoulos, E.B. [2009], An adaptive optimization scheme with satisfactory transient performance, Automatica, Vol. 45, No. 3 (2009), pp 716-723.

Kouvelas, A., Aboudolas, K., Kosmatopoulos, E.B. and Papageorgiou, M. [2011], Adaptive Performance Optimization for Large-Scale Traffic Control Systems, IEEE Transactions on Intelligent Transportation Systems, 2011, pp 1434 -1445.

Lin, C.J., Chang, C. and Hsu, C. [2004], A practical guide to support vector classification, Taiwan: National Taiwan University, 2004.

Lu, D., Kanchev, H., Colas, F., Lazarov, V. and Francois, B. [2011], Energy management and operational planning of a microgrid with a PV-based active generator for Smart Grid Applications, IEEE Transactions on Industrial Electronics, 2011.

Ma, J., Qin, S.J., Li, B. and Salsbury, T. [2011], Economic model predictive control for building energy systems, 2011 IEEE PES on Innovative Smart Grid Technologies (ISGT), 2011, 1-6.

Ma, Y., Borrelli, F., Hencey, B., Coffey, B., Bengea, S. and Haves, P. [2010], Model predictive control for the operation of building cooling systems, American Control Conference (ACC), 2010, 5106-5111.

Massoud A.S. and Wollenberg, B.F. [2005], Toward a smart grid: power delivery for the 21st century. IEEE Power and Energy Magazine, Vol. 3, no. 5 (2005), pp 34-41.

Mosteller, F. [1948], A k-Sample Slippage Test for an Extreme Population, The Annals of Mathematical Statistics, Vol. 19, No. 1 (1948), pp 58-65.

Moura, P.S. and De Almeida, A.T. [2010], The role of demand-side management in the grid integration of wind power, Applied Energy (Elsevier), Vol. 87, No. 8 (2010), pp 2581-2588.

Nghiem, T.X. and Pappas, G.J. [2011], Receding-horizon supervisory control of green buildings, American Control Conference (ACC), 2011, 4416-4421.

Oldewurtel, F., Parisio, A., Jones, C.N., Gyalistras, D., Gwerder, M., Stauch, V., Lehmann, B. and Morari, M. [2011a], Use of model predictive control and weather forecasts for energy efficient building climate control, Energy and Buildings (Elsevier), 2011.

Oldewurtel, F., Ulbig, A., Parisio, A., Andersson, G. and Morari, M. [2010], Reducing peak electricity demand in building climate control using real-time pricing and model predictive control, 49th IEEE Conference on Decision and Control (CDC), 2010, 1927-1932.

Oldewurtel, F., Ulbig, M., Morari, M., Andersson, G. [2011b], Building Control and Storage Management with Dynamic Tariffs for Shaping Demand Response in Electricity Grids, IEEE PES Innovative Smart Grid Technologies Europe. 2011.

Oyarzabal, J., Jimeno, J., Ruela, J., Engler, A. and Hardt, C. [2005], Agent based micro grid management system, 2005 International Conference on Future Power Systems, 2005.

PEBBLE Project [2010], Positive Energy Buildings thru Better controL dEcisions, 2010, website: http://www.pebble-fp7.eu.

Pichler, M., Droescher, A., Schranzhofer, H., Kontes, G.D., Giannakis, G.I., Kosmatopoulos, E.B. and Rovas, D.V. [2011], Simulation-assisted building energy performance improvement using sensible control decisions, 3rd ACM Workshop On Embedded Sensing Systems For EnergyEfficiency In Buildings (BuildSys11). 2011 (accepted).

Privara, S., Vana, Z., Gyalistras, D., Cigler, J., Sagerschnig, C., Morari, M. and Ferkl, L. [2011], Modeling and identification of a large multi-zone office building, 2011 IEEE International Conference on Control Applications (CCA), 2011, 55-60.

Qi, W., Liu, J. and Christofides, P.D. [2012], Distributed Supervisory Predictive Control of Distributed Wind and Solar Energy Systems, IEEE Transactions on Control Systems Technology, 2012, pp 1-9. 
Remund, J., Kunz, S. and Lang, R. [1999], METEONORM-Global meteorological database for solar energy and applied climatology, Solar Engineering Handbook, 1999.

Robbins, H. and Munroe, S. [1951], A stochastic approximation method, Annals of Mathematical Statistics, Vol. 22 (1951), pp 400-407.

Rokach, J.Z. [2012], Smart Houses in a World of Smart Grids, The Electricity Journal (Elsevier), 2012.

Sagerschnig, C., Gyalistras, D., Seerig, A., Privara, S., Cigler, J. and Vana, Z. [2011], Cosimulation for building controller development: The case study of a modern office building, CISBAT 2011. Lausanne, Switzerland, 2011.

Scholkopf, B., Smola, A.J., Williamson, R.C. and Bartlett, P.L. [2000], New support vector algorithms, Neural Computation, Vol. 12, No. 5 (2000), pp 1207-1245.

Scholkopf, B. and Smola, A.J. [2002], Learning with kernels, Citeseer, 2002.

Van Staden, A.J., Zhang, J. and Xia, X. [2011], A model predictive control strategy for load shifting in a water pumping scheme with maximum demand charges, Applied Energy (Elsevier), 2011.

Vapnik, V., Golowich, S.E. and Smola, A. [1996], Support vector method for function approximation, regression estimation, and signal processing, Advances in Neural Information Processing System,. 1996.

Wetter, M. [2011], Building Controls Virtual Test Bed, Lawrence Berkeley National Laboratory, 2011.

Zong, Y., Kullmann, D., Thavlov, A., Gehrke, O. and Bindner, H.W. [2012], Application of Model Predictive Control for Active Load Management in a Distributed Power System With High Wind Penetration, IEEE Transactions on Smart Grid, 2012, pp 1-8. 